Automation and Kobotics in Construction $X$

G.H. Watson, R.L. Tucker and J.K. Walters (Editors)

(C) 1993 Elsevier Science Publishers B.V. All rights reserved.

\title{
Site Layout of Construction Temporary Facilities Using Enhanced-Geographic Information System (GIS)
}

\author{
Min-Yuan Cheng a and James T. O'Connor ${ }^{b}$ \\ aDepartment of Construction Engineering \& Technology, National Taiwan Institute of \\ Technology, Associate Professor \\ bDepartment of Civil Engineering, University of Texas at Austin, Associate Professor
}

\begin{abstract}
This paper focuses on developing an automated site layout system for temporary construction facilities. The site layout of temporary facilities (TF) is an important preplanning task that can either enhance or adversely affect construction productivity. An efficient TF layout can significantly reduce construction conflicts and improve project efficiency. In this research, the system, ArcSite, was developed to assist the project manager in identifying suitable areas to locate TFs. ArcSite is comprised of a Geographic Information System (GIS) integrated with a database management system (DBMS). The system includes the knowledge specific to construction site layout, TF databases, Arc/Info databases, and GIS geoprocessing system. In the development of the system, industry rules of thumb and the state of the art in layout modeling were reviewed and compiled in a systematic form. ArcSite uses the concept of Searching by Elimination to generate the potential sites for each TF. A heuristic algorithm was developed to model the process of human decision-making and to identify the spatiai relationships between the different data layers which represent the site geographies. Through both qualitative and quantitative modeling of facility relationships, an objective function for calculating the Proximity Index (PI) was used to determine the optimal site of each TF. The primary features of the system are as follows: (1) integrate construction schedule and design information to lay out TFs for both short term and long term usage, (2) allow for the use of the $\mathrm{PF}$ as the TF and reallocation of space over time, (3) automatically generate potential sites for each TF, (4) identify optimal site, and (5) locate the TF within the optimal site. ArcSite improves the site planning efficiency by integrating spatial and thematic information into a single environment. Also, compared with current methods, ArcSite significantly improves the computational effort and increases the data accuracy and consistency. Therefore, this study achieves the goal of developing an automated information system to enhance the TF layout design.
\end{abstract}

\section{INTRODUCTION}

\subsection{Present Environment}

Owing to the competitive bidding structure of the construction industry, owners and bidding contractors are reluctant to spend the necessary money to cover the effective planning and management. While it is evident that the cost of site planning and construction of temporary facilities increases project overhead, unfortunately, there is little documented evidence that such an investment will improve overall project efficiency. Particularly, for contractors, the investment of the site planning is not viewed as critical. Therefore, for projects with tight budgets, it is easy to justify neglecting the planning of TF altogether. As a result, project managers generally do not have adequate staff or time to plan TF well. On 
construction sites, project managers usually accomplish the TF layout based on previous trialand-error and in an ad-hoc fashion. This was substantiated in a previous survey that found that most project managers layout TF based on experience, common sense, and adaptation of past layouts to present project [Rad 83]. Therefore, it is difficult to detect the layout problems until conflicts occur and relocation of temporary facility is required. Also, the inefficiency of correcting mistakes after-the-fact and dollars lost due to the lack of preplanning go undetected by management.

\subsection{Problem Statement}

Most of the project managers realize that the layout of a construction site has a considerable influence on the cost performance of the project. But how much influence? How can it be measured? There are no standard methods of measurement. Therefore, the manager can not quantify the productivity loss due to improper layout. Also, the manager can not evaluate the worth of good site layouts. Productivity loss is one of the main concerns in this study. Previous surveys show that productivity is lost in frequent worker travel on site for tools or materials, waiting for tools, material and other members of the crew, job interruptions, and working space conflicts. It should be realized that when conflicts occur and relocation of temporary facility is required, the loss is not only the cost of relocation of facility, but also includes time loss of waiting, job interference, labor morale and work discontinuity. This impact has a tendency to increase when the scope of project increases.

For the layout design, the project manager has to consider the characteristics and spatial constraints of the site and collect information from different resources such as A/E engineering, general contractors, subcontractors, and superintendents. Each party has his own interests and concerns. Therefore, the organization and analysis of the collected information as well as the decisions on tradeoffs between the interested parties have to be made by the project manager. After reviewing the problem area, the problems of TF site layout can be summarized as follows: (1). Design is based on incomplete and ill-structured information. (2). Site layout design depends on labor intensive manual approach. (3). Design relies heavily on individual experience. (4). There are diversity of solutions. (5). There is no exact rules or methods to follow. (6). Analysis lacks rigor: - dominant selection criteria, - driven almost exclusively by visual representations, - very difficult to link spatial features with thematic information, and appropriate tools to help users to record the previous arrangements. Based on the problems defined, this study focuses on the development of an automated and integrated computer system to solve the TF layout as an ill-structured problem. In addition to replacing the manual methods, the system produces TF layout solutions with the pre-defined levels of analytical rigor.

\subsection{Research Purposes and Objectives}

The primary purpose of this paper is to investigate the potential for automating the site planning tasks of locating temporary construction facilities. The sub-objectives required to achieve the primary objective are the following: (1) Gain the knowledge and experience of the experts in laying out the temporary facilities (TF); (2)Structure the knowledge and procedures for TF site layout in a systematic form; (3) Define the dominant variables and develop an evaluation method to determine the TF locations; and (4) Develop an automated site layout system using Geographic Information Systems (GIS) to implement the objectives mentioned above.

\subsection{Scope Definition}

In this study, the system, ArcSite, was developed to design the TF site layout. Using the same methodology and algorithms with appropriate modifications, AreSite can be applied to most construction sites and be suitable to different site locations, such as remote, suburban, and urban areas. The integration of construction schedules with both PFs and TFs enables ArcSite to lay out TFs for both short term and long term usage. 


\section{SYSTEM DEVELOPMENT}

\subsection{Knowledge Acquisition And Representation}

This section achieves the task of modeling the expert's knowledge and experience of site planning and expressing it in a systematic form. The procedures for modeling the experts' knowledge and experience for TF site layout were classified into three phases: (1) compiling the experts' knowledge and experience for TF site layout, (2) interpreting the knowledge into the knowledge base, and (3) translating the knowledge base into the ArcSite implementation forms (see Figure 1).

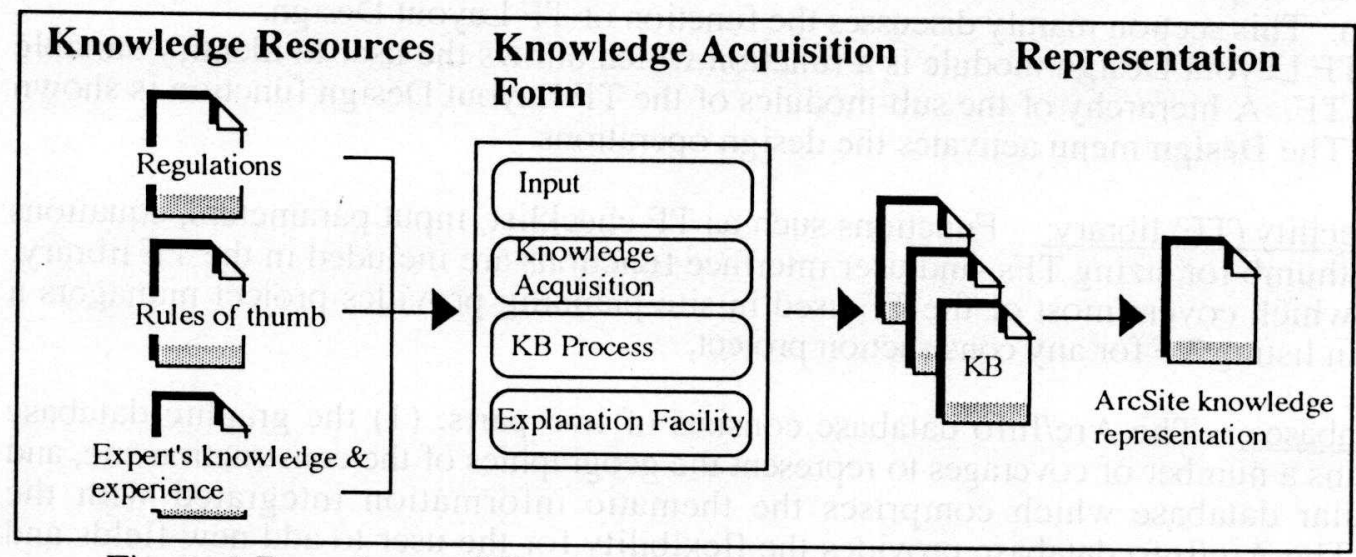

Figure 1 Procedures of Knowledge Acquisition and Representation

To develop the knowledge base, literature such as previous research work, and company handbooks were reviewed and knowledge was compiled according to three categories: (1) rules of thumb or experience of sizing TF, (2) TF design priority, and (3) regulations and safety standards.

After compiling the expert's knowledge and experience in site planning, the next task was to systematically interpret and express it into a knowledge base (KB). The knowledge base consists of four parts, viz., Input, Knowledge Acquisition, KB Process, and Explanation Facility. In the KB, Input is a mechanism designed to obtain input parameters to determine TF size and user inquiry. Knowledge Acquisition is a device to acquire the knowledge of site planning. The KB Process not only interprets the knowledge obtained in the knowledge acquisition phase into a systematic format, but also shows the process of reasoning. The Explanation Facility contains information which is not covered in the other three modules or advanced considerations and explanations required to improve the TF layout design.

Once the site layout knowledge base was established, it is necessary to translate the knowledge base into the ArcSite implementation forms suitable for analysis.

\subsection{System Usage}

System usage essentially involves identifying the potential users of the system. ArcSite can be used in three construction phases: (1) design, (2) pre-construction planning, and (3) during construction. During the design phase, ArcSite can be used to assist both experienced and inexperienced designers. Furthermore, since the layouts of PF and TF influence each other, designers must repetitively adjust and update the layouts as construction progresses. This iterative process can be accomplished efficiently using ArcSite. Before the construction commences, ArcSite can be used by project managers and superintendents to organize and analyze the information required for site planning. Using ArcSite, the spatial relationships between different data layers can be identified and criteria such as distance, adjacency, position, accessibility, and space can be evaluated to determine the areas in which the TF can be located. During construction, AreSite can assist the project managers and superintendents in 
tracking and updating the layout of the site by evaluating and recording the PF and TF construction schedules stored in the database.

\subsection{System Functions}

The functional modules of the system are developed according to the capabilities and information needed for the user to conduct the site layout design. After assessing the needs of the user, the requirements of the tasks are translated into system functions. A hierarchy of the system functions is shown in Figure 2. Six functional modules, namely File Manager, TF Layout Design, Spatial Analysis, Graphical Query, Database Query, and User and Program Interface are developed. The menus associated with each function are illustrated in the function menu column. This section mainly discusses the function of TF Layout Design.

The TF Layout Design module is a function which allows the user to identify suitabie areas for the TF. A hierarchy of the sub-modules of the TF Layout Design function is shown in Figure 3. The Design menu activates the design operations.

Temporary facility (TF) library: Functions such as TF checklist, input parameters, equations and rules of thumb for sizing TFs, and user interface functions are included in the TF library. The library which covers most of the TF used in site planning provides project managers a design base in listing TF for any construction project.

Arc/Info databases: The Arc/Info database consists of two parts: (1) the graphic database which contains a number of coverages to represent the geographies of the construction site, and (2) the tabular database which comprises the thematic information integrated with the coverages. The Arc/Info database provides the flexibility for the user to add new fields and attribute values into the tabular database.

Use of Permanent Facility (PF) as Temporary Facility (TF): The system allows for using a $\mathrm{PF}$ to temporarily support construction operations. A PF with a finish date earlier than the start date of the candidate TF and with a larger area than the required TF area can be used as a TF once approval from the user is obtained.

Design TF layout that changes over time: As construction progresses, some of the TFs are finished and removed from the site. Thus, the space occupied by the TF is available again. After evaluating the duration of TF on the site, the system deals with the flexibility of reallocation of space over time. Thus, the space occupied by the previous TF can be reutilized to locate successive TFs.

Potential site generation: Using the concept of Searching by Elimination, the system develops an algorithm for generating the potential sites for each TF. Considering the constraints and selection criteria, ArcSite identifies the spatial relationship between the data layers which represent the site geographies. The heuristic approach initiates searching the available space to locate temporary facilities, and then eliminates the areas occupied by PF, working areas adjacent to the PF, and the areas closed for safety consideration. A number of alternatives which satisfy the searching criteria are generated. In addition, ArcSite provides users the flexibility of changing searching criteria.

Identification of the optimal site: Through both qualitative and quantitative modeling of facility relationships, an objective function for calculating the Proximity Index (PI) was developed to rank the identified potential sites. The calculation of PI for each potential site is based on the four relationship matrices: PF/TF travel frequency matrix, TF/TF travel frequency matrix, PF/TF attract/repel matrix, and TF/TF attract/repel matrix. The PI is the sum of the product of the distance and relationship rating between facilities. The potential site with the minimal PI is the optimal site. In addition, ArcSite allows users to select their preferences of the potential sites. 


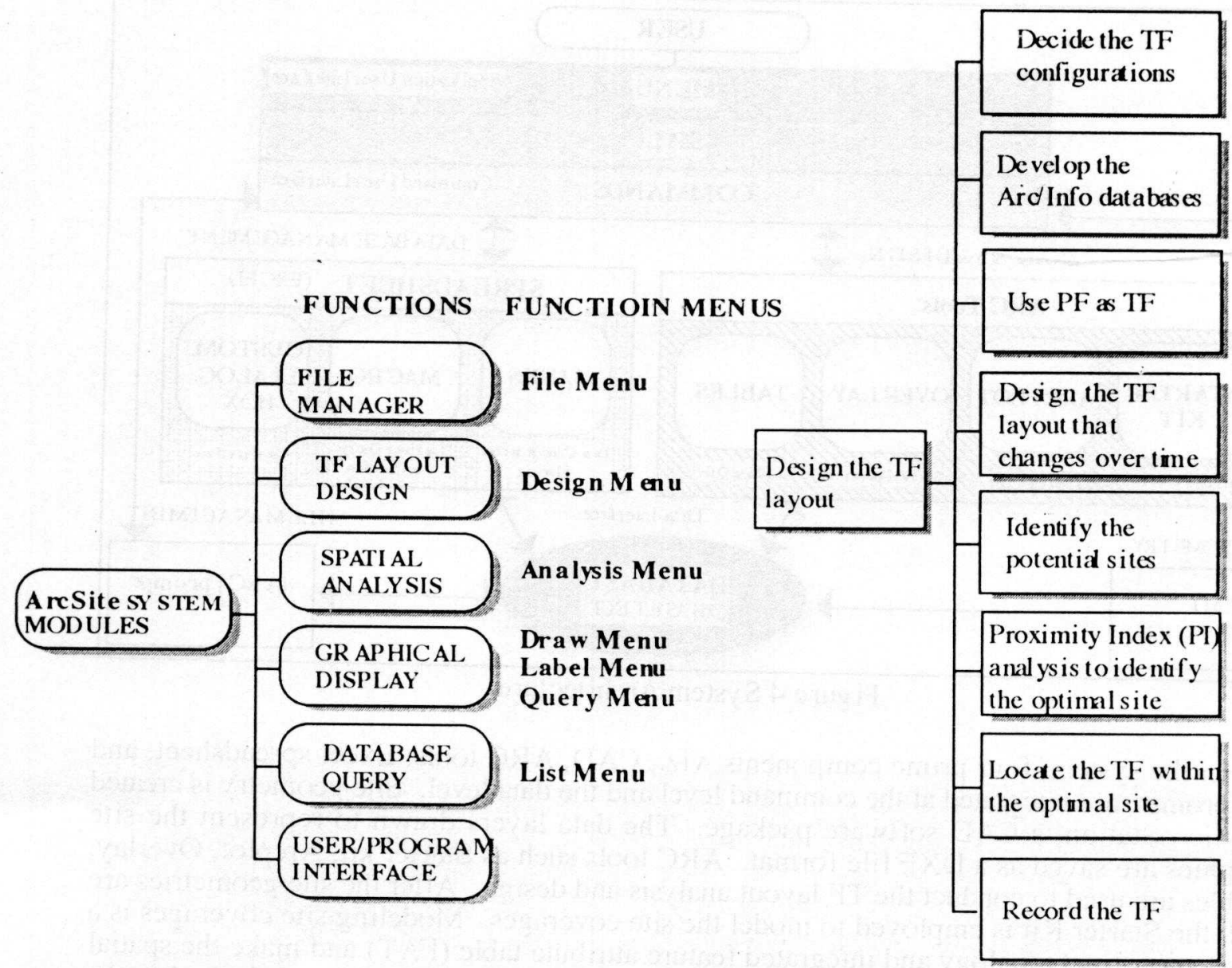

Figure 2 Hierarchy of System Functions

Figure 3 TF Layout Design Functions

Locate the TF within the optimal site: Usually, the area of the optimal site identified by AreSite is not equal to the size of the candidate TF. If the area of the optimal site is more than 1.5 times of the TF area, ArcSite locates a rectangle to represent the TF within the selected site. The functions included to locate the TF within the optimal site involve: (1) spatial operations to identify the vertices which intersect with the access roads, (2) identification of the optimal and second optimal vertices based on the PI rankings of the vertices, (3) generation of the base line based on the optimal and second optimal vertices, (4) user preference to decide the TF orientation, and (5) layout of the TF along the base line.

Record TF: The area assigned to the TF represents an occupied space which may need to be eliminated from the available space for the subsequent layout design. Thus, ArcSite dynamically generates a coverage to contain the TF and records the name of the created TF. Considering the PI analysis for the successive process, the coordinates of the TF are also recorded.

\subsection{System Architecture}

The tools used to develop the system functional modules are discussed in this section. Figure 4.4 shows the architecture of the system. Using SML, the user and program interface is integrated at three levels, namely application user interface, command user interface, and program data interface. 


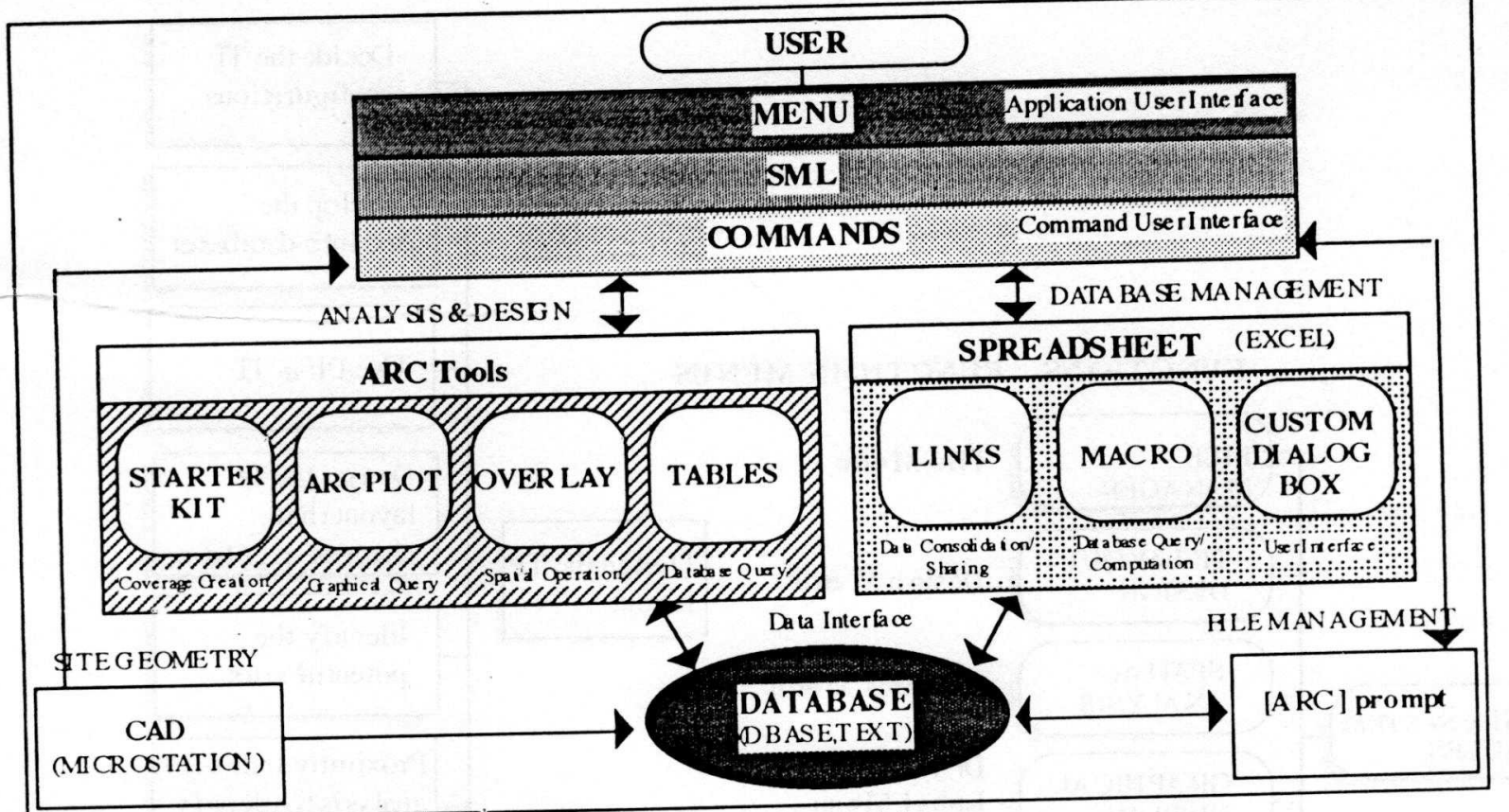

Fi gure 4 System Architec ture

In the system, four prime components, viz., CAD, ARC tools, Excel spreadsheet, and [ARC] prompt, are integrated at the command level and the data level. Site geometry is created using MicroStation, a CAD sof tware package. The data layers drawn to represent the site geographies are saved as a DXF file format. ARC tools such as Starter kit, Arcplot, Overlay, and Tables are used to conduct the TF layout analysis and design. After the site geometries are created, the Starter Kit is employed to model the site coverages. Modeling site coverages is a process to construct topology and integrated feature attribute table (FAT) and make the spatial data usable for analysis. Arcplot is used to perform the graphical query and display the analysis results. Overlay provides the basic operators for spatial analysis. Tables conducts the database query during the TF layout design. Using Excel spreadsheet, the functions of database management system such as adding of the tabular attributes, data consolidation and sharing, database query, and user interface are accomplished. Linking is useful for consolidating and sharing data from several worksheets and thereby designing an effective model. To automate the system commands for the worksheets to do database query and computation are recorded using macros. The custom dialog boxes are created using functions within Excel. Access to the [ARC] environment enhances the file management capabilities of the system. This component allow's the user to temporarily suspend the execution of the SML macros and exit to the [ARC] prompt to use the Arc/Info functions.

\section{SYSTEM RESULTS AND VALIDATION}

This study applied the data from the Bull Run Steam Plant (BRSP) project to ArcSite for testing the system. BRSP is a steam-electric generating plant consisting of four units of total 3.6 megawatts. It is located on the east bank of Melton Hill Lake in Anderson County, Tennessec. The result of the TF layouts generated by AreSite is shown in Figure 5 . Note the system identifies the optimal site of each TF on the site and provides the user with options to select his/her preference site. In addition, the TF layout solution is sensitive to the TF design sequence. Different TF design priorities can result in various layout solutions. Therefore, by changing the TF design sequence, the user can conduct "what-if" analysis to solve both real 
and hypothetical on-site design layout problems.

Figures 5 shows the layouts designed by AreSite. When comparing the layouts designed by ArcSite with the conventional approach, the reader will find that the two layouts are not identical. The layout discrepancies can be summarized into five reasons. First, ArcSite included not only the knowledge, information, and procedures stated in the BRSP technical report for TF site layout but also other experts' knowledge and information in the development of the system. Secondly, ArcSite might fail to represent all the factors that the project manager used in generating the layout, or perhaps the technical report did not include all the factors that the project manager considered in the development of the original TF layout. Thirdly, for practicality, this study clustered the TF with the same characteristics so that they would not clutter up large, open spaces on site that might be used for other purposes. The fourth difference relates to the diversity of the solutions. Often, it is difficult to tell which arrangement is best. As discussed in the problem statement, there are no standard guidelines or methods used to evaluate how good a site layout is. Finally, the knowledge and experience that the project manager brings to the TF site layout is nearly impossible to quantify. This represents a communication problem which can be classified in two phases: knowledge acquisition and representation. In the acquisition of knowledge, the training and expertise may lead different engineers to interpret data and terminology differently which can lead to differences of opinion on the job site. Regarding the realm of knowledge representation even on a heuristic level, there are many ways to represent the layout knowledge. It is difficult to ascertain if the knowledge representation is appropriate and complete.

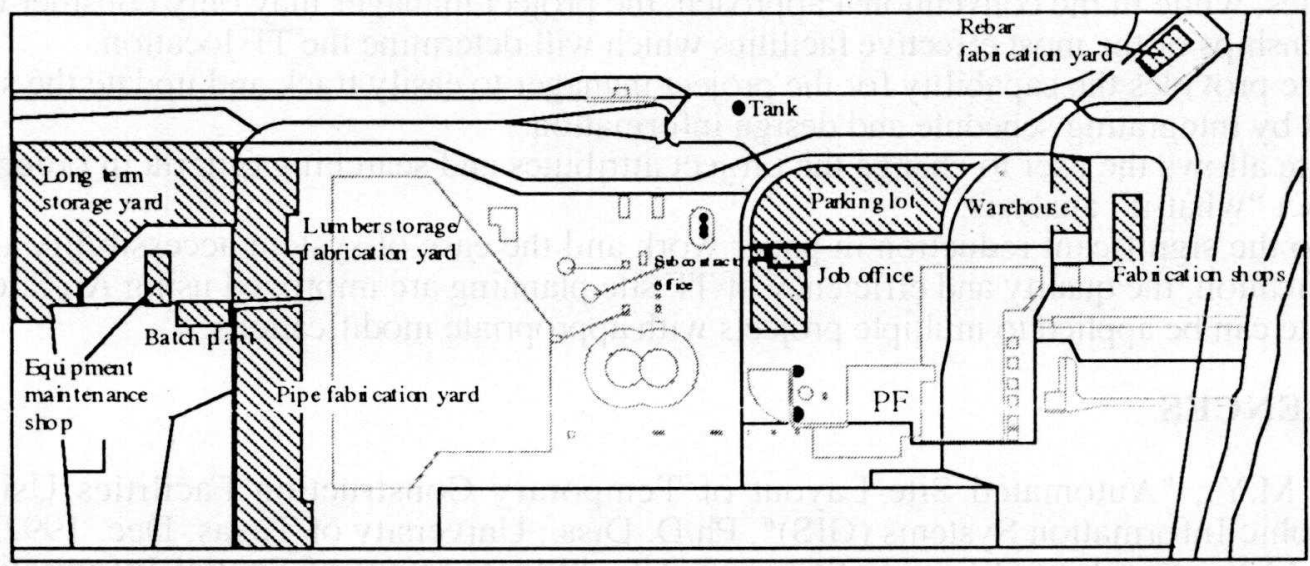

Figure 5 Final Temporary Facility Layout

\section{CONCLUSIONS}

The hypotheses of this research are validated by experimenting and implementing the system. Four conclusions are made as follows:

(1) TF layout problems can be effectively solved using a computerized system.

In the development of the system, the information required to select the optimal location for the TF was successfully represented and integrated in a computer environment. In addition, an automated site layout system was developed to identify the satisfactory solution for TF layout.

(2) GIS - Are/Info forms an effective foundation for TF site planning.

The basic functions of Arc/Info including information representation, spatial operations, graphical display and query, and adding functionality form an effective foundation for carrying out TF site layout. Using Are/Info, the investment of time and programming effort in developing the system was significantly reduced. 
(3) A robust system is required to solve the complicated TF layout problem. Over-simplified systems can result in unrealistic plans for actual projects.

ArcSite was developed to replace manual methods and assist planners in assessment the TF layout design. The system identified the rules of thumb and experience for sizing the TFs and used state of the art industry practices to lay down the TFs. Also, a heuristic approach for the facility layout problem was developed to eliminate the site choice of the TF as an illstructured problem. For practical site planning, ArcSite is a promising decision support system with a high utility value.

(4) The system improves both productivity and quality of site planning. follows:

The advantages of applying ArcSite in solving TF layout problem are identified as

- ArcSite systematically organizes the ill-structured information used for TF site layout.

- Owing to Arc/Info's ability to integrate locational and thematic information, ArcSite improves the site planning efficiency by eliminating the need for extracting data from various resources. Also, using ArcSite, the data integrity and accuracy are ensured.

- The computational information such as facility coordinates and distance between facilities are considered in ArcSite to lay out the TF.

- Compared with current methods, ArcSite significantly improves the computational effort and increases the data accuracy and consistency.

- ArcSite performs a series of complicated spatial operations and database queries to identify the optimal site. These tasks are difficult to perform manually.

- To identify the optimal site, ArcSite considers all of the relationships between the facilities, while in the conventional approach, the project manager may only consider the relationships of the most effective facilities which will determine the TF location.

- ArcSite provides the capability for the project manager to easily track and update the site layout by integrating schedule and design information.

- ArcSite allows the user to change the project attributes and searching criteria in order to conduct "what-if" analysis.

- Due to the significant reduction in paper work and the ease of system accessibility and modification, the quality and efficiency of TF site planning are improved using ArcSite.

- ArcSite can be applied to multiple projects with appropriate modifications.

\section{REFERENCES}

1. Cheng, M.Y., "Automated Site Layout of Temporary Construction Facilities Using Geographic Information Systems (GIS)", Ph.D. Diss., University of Texas, Dec. 1992.

2. Cheng, M.Y., Varghese, K., and O'Connor, J.T., "Management of Spatial Information for Construction Planning and Design Using Geographical Information Systems (GIS)" The 9th ISARC, Vol. 2, pp. 393-402, June 1992.

3. ESRI, 1989a "PC Arc/lnfo Reference Manual." Environmental Systems Research Institute, January 1989: Redlands, CA.

4. Handa, V. and Lang, B., "Construction Site Planning.", Construction Canada 88 05, pp. 43-49, 1988.

5. Hamiani, A., "ConSite: A Knowledge-Based Expert System Framework for Construction Site Layout" Ph.D. Diss., University of Texas, December 1987.

6. Neil, J.M., "Teaching Site Layout for Construction.", ASCE Mecting, Portland, OR, April 14-18, pp. 1-11, 1980.

7. Popescu, C., "Large Construction Site Design and Organization in Developing Countries.", SAE paper No. 780533, Society of Automotive Engineers (SAE), 1978.

8. Rad, P.F. and James, B.M., "The Layout of Temporary Construction Facilities.", Cost Engincering, Vol. 25, No. 2, pp. 19-27, A pril 1983.

9. Tommelein, I.D., "SightPlan: An Expert System that Models and Augments Human Decision-Making for Designing Construction Site Layouts.”, Ph.D. Diss., Stanford University, August 1989. 